\title{
Nanosilver fluoride as a caries arresting agent: A narrative review
}

Mohammed Zameer ${ }^{1} \bowtie$, (1) Sameen Badiujjama Birajdar ${ }^{2}$, (1) Syed Nahid Basheer ${ }^{3}$, Syed Wali Peeran ${ }^{4}$, (D) Syed Ali Peeran ${ }^{5}$, (1) Arun Reddy ${ }^{6}$

\section{Highlights}

The current paper foregrounds the effectiveness of a non-invasive treatment approach for dental caries using caries arresting agents.

\section{${ }^{1}$ M.D.S (Pediatric Dentistry)}

Registrar Pedodontist,

Armed Forces Hospital, Jazan, KSA

${ }^{2}$ General Dentist,

Sanjeevani Dental Clinic, Raichur, India

${ }^{3}$ Assistant Professor,

Department of Restorative Dental Sciences,

Jazan University, Jazan, KSA

4 Senior Registrar periodontist,

Armed Forces Hospital, Jazan, KSA

${ }^{5}$ Registrar Prosthodontist,

Armed Forces Hospital, Jazan, KSA

6 Associate Professor,

Department of Oral \& Maxillofacial

Orthodontics,

Navodaya Dental College, Raichur, India

\section{Correspondence:}

Department of Pediatric Dentistry, Armed Forces Hospital, Jazan, KSA E-mail address:

drmohammedzameer@gmail.com
The newer nanotechnology-based caries arresting agent presents effective antibacterial properties against cariogenic bacteria and modulation of the demineralization-remineralization balance for teeth.
The silver nanoparticle-based preparations present to overcome the limitations of using silver ion-based solutions for caries arrest.

\section{Abstract}

Dental caries is the most prevalent oral disease that continues to affect almost every country in the world. The contemporary management of dental caries focuses on non-restorative, non-invasive, and micro-invasive treatment approaches that arrest or reverse the caries process at a lesion level and reduce the loss of sound tooth structure. One of these approaches is the employment of caries arresting agents that possess antibacterial and remineralizing properties. Silver diamine fluoride (SDF) has drawn significant attention as an efficient caries arresting agent in children and adults. The major limitation with the use of SDF is the dark black staining of the carious tissue that compromises the esthetics. Silver ions are known for their antimicrobial effects, and silver nanoparticles (AgNPs) have the added advantage that it increases the surface area for exposure to the microbes. Literature reports that AgNPs have antimicrobial potential against predominant cariogenic flora. It has led to the development of nanosilver fluoride (NSF), a new colloid based on AgNPs, chitosan, and fluoride. It has shown to overcome the clinical limitations of SDF as it does not cause carious lesion staining. However, the current scientific literature lacks a comprehensive review of the benefits of using NSF for caries prevention and arrest. Thus, the purpose of this paper was to review the studies and clinical trials on NSF as a caries arresting agent, including antibacterial actions and modulation of the demineralizationremineralization balance.

Keywords: Dental Caries; Fluoride; Tooth Demineralization

Received: 27 March 2021

Accepted: 05 May 2021

Online First: 05 May 2021 


\section{INTRODUCTION}

Dental caries is the most prevalent oral disease that continues to affect almost every country in the world. ${ }^{1}$ The contemporary dental practice focuses on non-restorative, non-invasive, and micro-invasive treatment approaches that arrest or reverse the caries process at a lesion level and reduce the loss of sound tooth structure. These treatment approaches include the employment of caries arresting agents, sealant, resin infiltration, fluoride varnish, fluoride toothpaste, and gel. ${ }^{2-4}$

Silver diamine fluoride (SDF), a metal ionbased topical fluoride solution, has drawn significant attention as an efficient caries arresting agent in children and adults. ${ }^{5-8}$ Studies ${ }^{9-13}$ have reported its effectiveness against cariogenic bacteria and fungi, and its remineralizing potential on enamel and dentin. ${ }^{13-16}$ Major limitation with the use of SDF is the dark black staining of the carious lesions due to the precipitation of silver particles on the carious tissue. ${ }^{17,18}$ Hence, its use in the aesthetic zone is not encouraging. ${ }^{19-21}$ The other limitations of SDF use include; metallic taste, short-term staining to the skin which resolves in 2 to 14 days $^{22}$ and mildly painful lesions on accidental contact of SDF solution with oral mucosa which generally heal within a couple of days. ${ }^{6,23}$ To counter the undesirable staining, it is suggested to follow a combination protocol; potassium iodide (KI) application immediately after the use of $\mathrm{SDF}^{17,24}$ or SDF mixed with glutathione(GSH) bio-molecule. ${ }^{25}$

The investigations on following the combination protocol revealed a positive effect in reducing the staining when compared to the use of SDF alone. ${ }^{25-27}$ However, the use of KI has been associated with poorer caries control ${ }^{28}$ and a certain degree of staining that can compromise the esthetics is observed in the carious arrested lesions. ${ }^{24,26,29,30}$ Furthermore, the use of $\mathrm{KI}$ is contraindicated in pregnant women and during the first-six-months of breast-feeding because of the concern of overloading the developing thyroid with iodine. ${ }^{22}$

The advancement in nanotechnology led to the development of silver nanoparticles (AgNPs). The antibacterial properties of AgNPs have been well recognized in the medical field. ${ }^{31,32}$ These particles are assumed more efficient due to their greater surface area that would increase the contact with microbial cells. ${ }^{29}$ AgNPs have drawn attention from the dental researcher for their antibacterial potential that can be utilized in anticaries approaches. ${ }^{33}$

Table 1 summarizes history and chronology of various silver compounds for caries management. Literature $^{12,34}$ reports that AgNPs have antimicrobial potential against predominant cariogenic flora. Furthermore, researchers have explored the combination of AgNPs and fluoride to include the advantages of each individual component.

Nano-silver fluoride (NSF), a new colloid based on AgNPs, chitosan, and fluoride was developed as a caries arresting agent that comprises both antibacterial and remineralizing properties. ${ }^{30}$ Hence, NSF is a promising agent as it overcomes the clinical limitations of SDF as it causes no carious lesion staining. 29,30,35,36 This is due to the size of silver particles and also because the nanoparticles do not undergo oxidation. ${ }^{29}$ This new formulation is safe for use in humans, and controlled clinical trials have shown its anticaries property. ${ }^{36-38}$ However, the current scientific literature lacks a comprehensive review of the benefits of using NSF in the treatment of dental caries. Thus, the purpose of this paper is to review NSF as a caries arresting agent, including antibacterial actions and modulation of demineralization-remineralization balance. 
Table 1. History and chronology of various silver compounds for caries management

\begin{tabular}{ll}
\hline Year & Brief Description \\
\hline $1846^{52}$ & The first recorded use of Silver Nitrate (SN) in the treatment of dental caries. \\
\hline $1891^{53}$ & $\begin{array}{l}\text { Reports have been made by Stebbins on the use of SN for dental caries inhibition. It has also been } \\
\text { hypothesized that caries inhibition was due to bacterial-killing and the formation of a "black crust," which is a } \\
\text { sclerotic protective coating of secondary dentin. }\end{array}$
\end{tabular}

$1902^{52} \quad$ The first attempt has been made by Szabo to examine the penetration of SN in the dentinal tubules. It was found that $\mathrm{SN}$ penetrates through the whole carious lesion and into the sound dentin tissue. This penetration was not more than $0.5 \mathrm{~mm}$.

$1905^{54} \quad$ Miller proposed dentin protection against acids with the use of SN.

$1917^{55} \quad$ The first research director at Forsyth Institute in Boston, Perce Howe presented the modification of silver which was reduced from the solution with formalin and recommended its use for sterilization of the disintegrated dentin over the pulps. The solution penetrated the whole affected dentin but did not penetrate sound tooth tissue. He stated that the coagulation of the content of dentinal tubules gives dentin protection against the acids. After this, a renaissance began in the clinical use of SN.

1920s to $1930 \mathrm{~s}^{52} \quad$ 'Howe solution' is used as a disclosing agent and bactericide in cavity preparation.

$1941^{52} \quad$ Zander HA published a histological investigation showing contrary results with the use of SN; The use of SN solution as a disclosing agent in dentin is considered doubtful. Because the bacteria were seen penetrating almost twice as far as the $\mathrm{SN}$ into the dentin. The use of $\mathrm{SN}$ as a disclosing agent for caries in enamel is of great value as it arrests caries for some time.

1940s to $1970 s^{56} \quad$ Five studies were published on the use of SN in the management of caries. Three of these studies showed no significant reduction in the incidence of caries when compared with no treatment. The other 2 studies evaluated caries arresting effect of $\mathrm{SN}$ solution.

$1970 \mathrm{~s}^{53,57} \quad$ Developed silver diamine fluoride (SDF) to combine the actions of silver and fluoride. The combination showed its effects on the prevention and arrest of dental caries. This led to the approval of first SDF product, Saforide, Japan.

$1980 s^{58-61}$

The inhibitory effect of the combination of Silver and Fluoride on oral bacteria was assessed. Silver ion was shown to be the major inhibitor of oral bacteria. In clinical trials, another combination protocol was examined, using Silver fluoride followed by Stannous Fluoride(SnF2). It showed to have synergistic effects in arresting caries lesions. Further, this combination had no adverse effect on permanent successors.

$1990 \mathrm{~s}^{8}$

SDF has been widely used in Brazil and Australia with in-vivo studies carried out in the respective countries to prevent and arrest dental caries.

2001 to

$20136,11,16,49,62-69$
The anti-carious activity of SDF was investigated in several in-vitro studies. Hence SDF was documented to have antibacterial effects and remineralizing properties. Multiple randomized controlled clinical trials were carried out during the same time to evaluate the efficacy of SDF in preventing dental caries. It was found to be an effective non-invasive treatment approach in preschool children and schoolchildren. Clinical trials also document SDF to arrest root caries and prevent new root caries formation in elderly people.

$2014^{22}$ The Food and Drug Administration(FDA) cleared SDF for use in the United States of America as a desensitizing agent.

2016 22 SDF's off-label use was legally permissible and a CDT code had been approved for caries arresting medicaments to facilitate documentation and billing. The UCSF School of Dentistry Paradigm Shift Committee formed a subcommittee and developed a standardized guideline, protocol, and consent for the use of SDF. 
Table 1. Continued

2009 to $2019^{33,70}$

Researchers investigated AgNPs as an antimicrobial agent and confirmed it to inhibit the growth of cariogenic bacteria and biofilm adhesion. They were also found to preserve the collagen matrix and impede demineralization of enamel and dentin. AgNPs have been incorporated into dental materials for caries control. Sodium Fluoride $(\mathrm{NaF})$ was combined with AgNPs to prevent and arrest caries. AgNPs were added to restorative materials such as restorative resin and adhesive systems with an intention to prevent secondary caries. Furthermore, AgNPs have been utilized in orthodontics accessories such as brackets, elastomeric ligatures, adhesives, and removable retainers.

2014 to $2020^{29,30,35,48}$ Multiple studies have investigated the antibacterial properties of NSF. The AgNPs in the formulation have the added advantage that it increases the surface area for exposure to the microbes. It has shown to inhibit cariogenic bacterial growth and biofilm adhesion and cause bactericidal actions without harming human cells. Studies confirm effective remineralizing properties of NSF on both the primary and permanent tooth. NSF has shown to be a simple, inexpensive, non-toxic, non-invasive caries arresting agent, and it did not present carious lesion staining.

\section{Mechanism of action of NSF}

The antibacterial properties of nanomaterials have been investigated, and the antibacterial effect showed to come from AgNPs. Although the exact mechanism of antibacterial action of AgNPs has not been entirely understood, several antibacterial actions have been proposed and elaborated in Figure 1. Chitosan was added to the AgNPs as it acts as a carrier and stabilizes the compound. Further, to make this a more comprehensive agent, fluoride was added to the AgNPs-chitosan compound to fortify the antibacterial properties and prevent demineralization. This new formulation, called NSF, has been reported for caries prevention and arrest. ${ }^{33}$

\section{Antibacterial properties of NSF}

Streptococcus mutans (SM) are the primary cariogenic bacteria, and they are associated with the initiation and progression of carious lesions. The oral bacteria exist collectively in the extracellular matrix to form a biofilm, which increases the resistance of microorganisms to antibacterial agents. Table 2 summarizes five in vitro studies and one clinical trial investigating the antibacterial effect of NSF on cariogenic bacteria.

Studies 39,40 have shown that the antibacterial activity of AgNPs increases with a decrease in their particle size. Accordingly, few studies have shown AgNPs in the range of $2.56 \pm 0.43 \mathrm{~nm}, 3.2$ $\pm 1.2 \mathrm{~nm}$ and $5.9 \pm 3.8 \mathrm{~nm}$ in NSF formulations to favour the antibacterial activity against
SM. ${ }^{35,38,41}$ Sodium borohydride has been regularly used as a reducing agent in preparing NSF formulations. ${ }^{38,41,42}$ However, due to concerns over toxicity, some researchers have used thiolated polyethylene glycol (PEG) as both a reducing agent and a capping agent for its preparation. Several advantages have been reported for the use of PEG; it increases AgNPs stability to a level that they can be preserved at high ionic concentrations, PEG-coated AgNPs have shown to be less toxic than those with other capping agents and are less liable to oxidize. ${ }^{35}$ Comparison between NSF and SDF for minimum inhibitory concentration (MIC) and minimum bactericidal concentration (MBC) values showed better results with the NSF. Cytotoxicity assessment by hemolytic activity showed NSF to be less toxic to human erythrocytes than SDF. Another study by Yin et al. ${ }^{35}$ showed halfmaximal inhibitory concentration (IC50) of PEGAgNPs against SM to be half of IC50 against human gingival fibroblasts (HGF-1). This indicates the provision of bactericidal action without harming human cells. NSF showed greater anti-adherence and anti-acidogenicity effects against $\mathrm{SM}$ when compared to sodium fluoride $(\mathrm{NaF}) .{ }^{43} \mathrm{NSF}$ has been suggested as an effective SM biofilm inhibitor because it has shown to reduce the CFU counts and dental biofilm inhibition values. ${ }^{30,42-44}$ Thus, NSF formulation can act as a more biocompatible antibacterial agent against SM. 


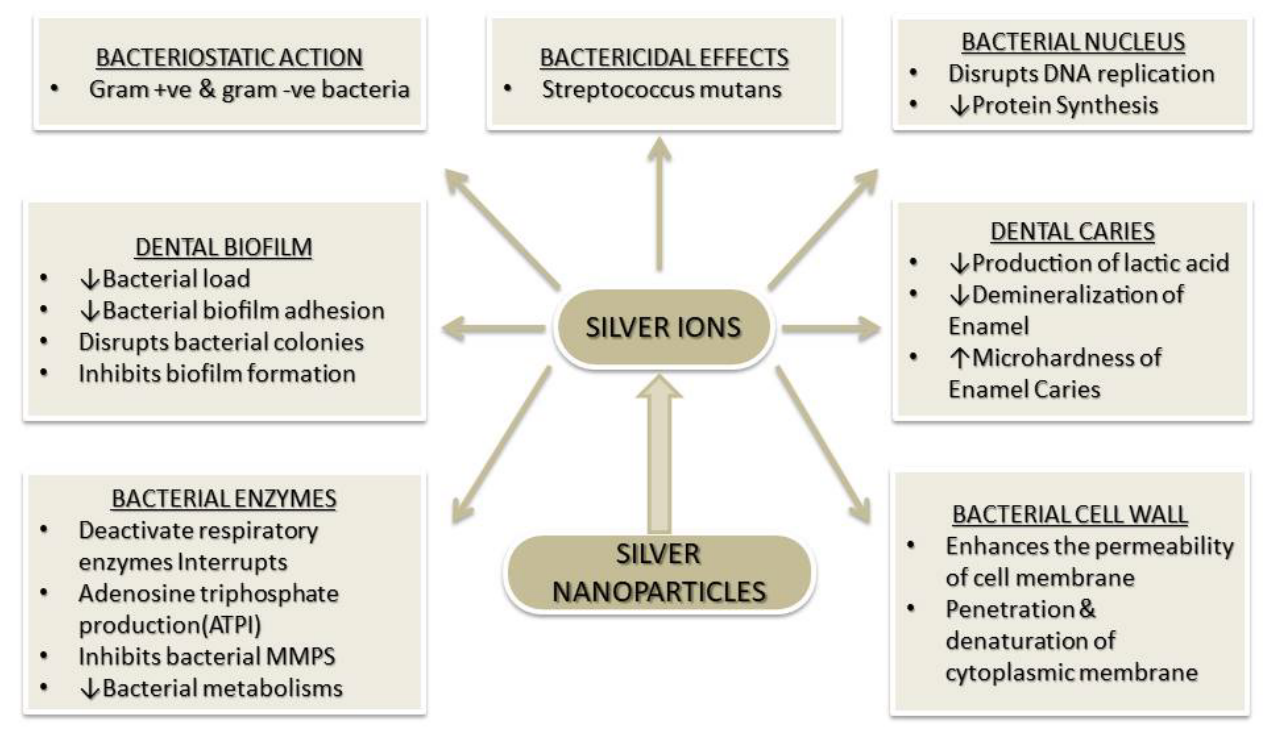

Figure 1. Mechanism of action of silver nanoparticles

\section{Remineralizing properties of NSF}

Table 3 summarizes seven laboratory studies that have evaluated the NSF effectiveness in remineralizing enamel and dentin of primary and permanent teeth. NSF formulation was compared with $\mathrm{NaF}$ for primary tooth enamel remineralization and it showed remineralizing effect similar or greater to NaF.42,43,45,46 In a study by Akyildiz et al. ${ }^{47}$ comparison of NSF with SDF and $\mathrm{NaF}$ showed that all the three remineralizing agents were statistically significant in rehardening the artificially demineralized enamel specimens of third molars. However, NSF was not shown to be as effective as $\mathrm{SDF}$ and $\mathrm{NaF}$. SDF $(12 \%)$ and NSF formulation $(2.5 \% \mathrm{NaF}$ with PEG-AgNPs) have shown similar effects for dentine caries remineralization and collagen degradation inhibition. ${ }^{48}$ In a study by Sayed et al. ${ }^{30}$ SDF has shown to cause distortion in the dentin collagen morphology but provides an intrafibrillar pattern of mineral deposition. However, NSF showed to preserve collagen structural morphology and provides intrafibrillar remineralization. The change in the collagen fiber morphology has been related to the high $\mathrm{pH}$ of $\mathrm{SDF}(\mathrm{pH} 10-12)$ compared to NSF ( $\mathrm{pH} 8-9$ ). This indicates better biocompatibility with the use of NSF and it can be an alternative agent for SDF.

\section{Clinical trials on the effectiveness of NSF in arresting dental caries}

Table 4 summarizes four clinical trials that investigated the effectiveness of NSF formulations in preventing and arresting caries in children. Tirupathi et al. ${ }^{36}$ have compared caries arresting efficacy of NSF preparation and SDF on an annual application in primary molars. It showed a $77 \%$ success rate with NSF preparation compared to $71.05 \%$ success in the SDF group. Although the success rate was comparatively better in the NSF group than the SDF group, the difference was not statistically significant. In a study by Santos et al..$^{38}$, NSF formulation has shown the results $(66.7 \%)$ of caries arrest similar to that of SDF as reported in the clinical trial by Llodra et al.(6) (>55\%) but was lower than the outcome reported by Chu et al. ${ }^{49}(>96 \%)$ This was due to difference in the trial design and intervals of application. However, they both have shown to be effective in arresting dental caries. ${ }^{38}$ Another study by Nagireddy et al. ${ }^{50}$ has shown 65.21\% effectiveness with NSF formulation in arresting caries after 12 months of follow up; this was comparable to $67 \%$ NSF results reported in the clinical trial by Santos et al. ${ }^{38}$. NSF application is a simple, inexpensive approach that requires no comprehensive dental clinical setup and can be affordable by most communities. 
Table 2. Studies on antibacterial properties of nanosilver fluoride

\begin{tabular}{lll}
\hline $\begin{array}{l}\text { Study and } \\
\text { its design }\end{array}$ & Characteristics and Summary Intervention & Study Findings \\
\hline
\end{tabular}

$\begin{array}{ll}\text { Sayed M et al, 202030 } & \text { 1) Sample: } 25 \text { demineralized dentin specimens } \\ \text { In-vitro study } & \text { 2) Distribution: } 5 \text { groups ( } \mathrm{n}=5) \text {; i) SDF, ii) AgNPs/KF, } \\ \text { Test Microbe: SM } & \begin{array}{l}\text { iii) KF, iv) AgNPs, v) Control. } \\ \text { 3) Outcome measures: i) Measurement of regeneration } \\ \text { potential of SM through CFU values, ii) Assessment of } \\ \text { bacterial growth inhibition through optical density }\end{array}\end{array}$

NSF preparation (AgNPs/KF) has shown to possess effective antibacterial properties

\begin{abstract}
Yin IX et al, $2020^{35} \quad$ 1) Antibacterial and cytotoxic effect of PEG-AgNPs In-vitro study against $\mathrm{SM}$ and $\mathrm{HGF}-1$ vs $\mathrm{AgNO}_{3}$ (comparator), and SDF (positive control) through IC50 values
\end{abstract}

Test Microbe: SM
2) Antibacterial effects (IC50 values) of 4 fluoridated PEG-AgNPs solutions containing various ratios of PEGAgNPs (12800, 6400, 1600, and 400 ppm Ag) and $\mathrm{NaF}$ (fixed at $2.5 \%=11,310 \mathrm{ppm} \mathrm{F}$ )
PEG-AgNPs have shown to inhibit the growth of SM.

Solution with the combination of $\mathrm{NaF}$ and PEG-AgNP showed no synergistic antibacterial effect but provides a therapeutic window for fine-tuning of each component's ratio and concentration to inhibit SM without harming the human cells.

\begin{abstract}
Silva et al, $2018^{43}$
In-vitro study
\end{abstract}

Test Microbe: SM
1) Sample: 27 enamel fragments of primary teeth.

2) Distribution: 3groups(n=9); i) NSF (Experimental group), ii) $\mathrm{NaF}$ (positive control), iii) Deionized water(negative control)

3) Outcome measures: i) Acidogenicity - $\% \mathrm{pH}$ variation,

ii) Quantification of bacterial adherence through CFUs and subsequent $\%$ bacterial adsorption inhibition.
NSF was found more effective in preventing $\mathrm{pH}$ decline and bacterial adhesion in comparison with $\mathrm{NaF}$.

\section{Teixeria et al, $2018^{42}$ \\ In-vitro study \\ Test Microbe: SM \\ 1) Distribution: 3groups; i) NSF containing dentifrices (test dentifrice), ii) $\mathrm{NaF}$ containing dentifrices (positive control), iii) Deionized water (negative control). \\ 2) Outcome measures: i) MIC, ii) MBC, iii) \% bacterial adsorption inhibition, iv) Acidogenicity - $\% \mathrm{pH}$ variation}

NSF containing dentifrices have shown better antibacterial effect compared to $\mathrm{NaF}$ containing dentifrices.

\section{Freire PLL et al, $2017^{44}$ \\ A randomized, pilot clinical trial study}

Duration:

Test Microbe: SM

Targino et al, 201441

In-vitro study

Test Microbe: SM
1) Sample: 12 schoolchildren of both genders, aged:78yrs.

2) Distribution: 2groups; i) NSF, ii) control (saline solution).

3) Outcome measures: i) Biofilm pH measurement, ii) Bacterial growth measurement by spectrophotometry, iii) OHI-S, iv) Bacterial count through CFU.
NSF has shown to reduce SM growth and presented as an effective means to inhibit dental biofilm formation.
1) NSF vs [CHX and SDF] (Control solutions)

2) Outcome Measures: i) Antibacterial activity through MIC and MBC ii) Cytotoxicity through hemolytic activity in human erythrocytes.
NSF showed as an effective antimicrobial agent similar to SDF. NSF has shown lower toxicity to living cells when compared to SDF. 
Table 3: In vitro studies on remineralizing properties of nanosilver fluoride

Study and its $\quad$ Characteristics and Summary Intervention
design

Sayed M et al, $2020^{30}$

1) Sample: 25 Dentin specimens from bovine incisor roots.

2) Distribution: 5 groups $(n=5)$; i) $\mathrm{SDF}$, ii) $\mathrm{AgNPs} / \mathrm{KF}$, iii) $\mathrm{KF}$,

iv) AgNPs, v) Control.

3) Outcome measure: Transmission electron microscopy

Zhao IS et al, $2020^{48}$
1) Sample: Extracted sound third molars.

2) Distribution: 18 dentine slices cut into 54 blocks which were divided into 3 groups(n=18); i) Group 1 - SDF, ii) Group 2 PEG-AgNPs with NaF, iii) Group 3 - Deionized water.

3) Outcome measures: i) SEM: surface morphology and crosssectional features, ii) Spectrophotometry: collagen degradation, iii) XDR: crystal characteristics.
NSF preparations have preserved dentin collagen, whereas, SDF has shown to alter dentin collagen morphology
$\mathrm{NaF}$ solution with PEG-AgNPs showed a similar effect to SDF in remineralizing artificial dentine caries and inhibiting collagen degradation.
Akyildiz et al, 201947
1) Sample: 45 humans third molars
2) Distribution: 180 enamel specimens were divided into 4groups; i) NSF, ii) SDF, iii) NaF, iv) control.
3) Outcome measures: i) VHN, ii) SEM.

NSF was not found comparatively effective as $\mathrm{NaF}$ varnish and SDF on enamel caries lesions.
Silva AV et al,
1) Samples: 33 primary tooth specimens
$2019^{45}$
ii) $\mathrm{G} 2-\mathrm{NaF}$ (positive control), iii) G3-No remineralizing agent(negative control)
2) Distribution: 3groups ( $n=11)$; i) G1-NSF(experimental),
3) Outcome measure: OCT

Teixeria et al, $2018^{42}$
1) Sample: 48 primary molars

2) Distribution: 3groups ( $n=16$ units); i) NSF containing dentifrices (test dentifrice), ii) $\mathrm{NaF}$ containing dentifrices (positive control), iii) Deionized water (negative control).

3) Outcome measures: Microhardness test, OCT
$\mathrm{NSF}$ is as efficient as $\mathrm{NaF}$ for enamel remineralization in primary teeth.

\begin{abstract}
NSF is as effective as $\mathrm{NaF}$ for enamel remineralization in primary
\end{abstract} molars.
Silva et al, $2018^{43}$
1) Sample: primary tooth enamel fragments.
2) Distribution: 33 samples into 3 groups $(n=11)$; 1$)$
$\mathrm{NaF}$ (positive control), ii) NSF (experimental group), iii)
Deionized water(negative control)
3) Outcome measures: Microhardness test, Fluorescence spectroscopy and OCT

\begin{abstract}
NSF is as efficient as NaF for primary tooth enamel remineralization.
\end{abstract}
Nozari A et al, 201746
1) Sample: 80 sound primary anterior teeth
2) Distribution: 60 samples into 4 groups $(n=15)$; i) NSF, ii)
$\mathrm{NaF}$ varnish, iii) n-HAP, iv) Control(no treatment)
3) Outcome measures: SMH, AFM

NSF can have greater remineralizing capability than $\mathrm{NaF}$ varnish and n-HAP for primary anterior teeth

SDF: Silver diamine Fluoride; NSF: Nanosilver fluoride; AgNPs: Silver nanoparticles; KF: Potassium fluoride; PEG-AgNPs: polyethylene glycol-coated silver nanoparticles; NaF: Sodium fluoride; SEM: scanning electron microscopy; XDR: X-ray diffraction; VHN: Vickers microhardness; OCT: Optical coherence tomography; n-HAP: Nano-Hydroxyapatite serum; SMH: Surface microhardness; AFM: Atomic Force Microscopy. 
Table 4. Clinical trials assessing the effectiveness of nanosilver fluoride in arresting dental caries

Study

Characteristics and Summary Intervention
Study Findings

NSF has shown to be an anticaries agent and presents a non-invasive option for arresting dental caries in children
Nagireddy VR et al, 1) Sample: 60 children of the 4-9years age range $2019^{50}$

Duration:1year
2) Distribution:100 primary molars were divided into 2 groups;

i) NSF - experimental group, ii) Saline - control group

3) Follow up: after 7days, 5months and 12months
An annual application of NSSF showed similar efficacy as SDF in arresting dentinal caries in primary molars.

Duration:1year

2) Distribution: 159 primary molars were divided into 2 groups.

i) NSSF - experimental group, ii) SDF - positive control

3) Follow up: 1month, 3months, 6months and 12months.
An annual application of NSF solution has shown to be effective in hardening and arresting dentine caries in primary teeth.
Burns J, Hollands K, 1$)$ Sample: 60 children with $6.31( \pm 0.60)$ mean age range $2015^{37}$

Duration:1year
2) Distribution:130 primary molars were divided into 2 groups;

i) NSF - experimental group, ii) Saline - control group

3) Follow up: 1week, 5month, 1year
NSF has shown to be an effective caries arresting agent for children in poor communities.

\section{of $6.31 \pm 0.60$ years.}

Duration:1year
2) Distribution: 130 primary teeth were divided into 2 groups( $n=65)$.

i) NSF - experimental, ii) Water - control

3) Follow up: 1week, 5months, and 12months.

NSSF: Nanosilver incorporated sodium fluoride; SDF: Silver diamine Fluoride;

\section{NSF vs SDF on caries lesion staining}

Table 5 summarizes 4 in-vitro studies assessing the staining effects of NSF. The staining phenomenon with SDF application is due to the silver particle's precipitation and their oxidation on the carious dentin. ${ }^{17,18}$ Contrary to this, NSF formulations do not form oxides when coming in contact with the medium's oxygen and hence does not cause dentin staining. ${ }^{38}$ The study by Sayed et al. ${ }^{30}$ showed that no colour change observed over time with the reduction in the size of AgNPs. Another study by Espíndola-Castro et al. ${ }^{29}$ showed that 2 weeks after the NSF application, yellowish stains were seen on the teeth. The chitosan in the composition of NSF formulation is believed to be the cause of this stain formation. However, these stains were removed later by toothbrushing, and the tooth colour restored to the baseline values. The authors also observed that this staining could also be removed easily with a gauze. The precise quantification of different colour characteristics carried out in other studies with an objective instrumental measurement approach. It revealed that fluoridated polyethylene glycol-coated silver nanoparticles (PEG-AgNPs) showed no observable dentin staining. The dentin discolouration displayed with SDF application was not acceptable clinically as it was more than ten times higher than the acceptable upper threshold. 35,48,51 Therefore, the NSF formulations have been suggested as a better alternative for caries arrest to overcome the staining disadvantage of SDF. 
Table 5. Nanosilver fluoride and silver diamine fluoride

\section{Study Characteristics and Summary Intervention Study Findings}

Espíndola-Castro 1) NSF formulations(600 and 1500ppm) vs other commercially et al $2020^{29}$ available cariostatic agents (Advantage arrest, Riva star and cariestop)

2) Sample \& Distribution: 75 extracted human molars were divided among 5 cariostatic agent $(\mathrm{n}=15)$

3) Spectrophotometric analysis: i) Before application, ii) After 2weeks, iii) After 4weeks.
NSF formulations can be an alternative to SDF as they do not compromise esthetics.

\section{Sayed $\mathrm{M}$ et al, 1) $\mathrm{SDF}$ vs KF vs AgNPs vs AgNPs/KF(Nano silver/potassium $2020^{30}$ fluoride) \\ 2) Sample \& Distribution: 100 bovine dentin specimens and divided among 5groups i) SDF, ii) AgNPs/KF, iii) KF, iv) AgNPs, v) Control. \\ 3) Spectrophotometric analysis: i) Before surface treatment, ii) Immediately after surface treatment and after, iii) 1 day, iv) 2 days, v) 7 days.}

\author{
NSF preparation can be an \\ alternative to SDF as it shows \\ no colour change over time
}

Yin IX et al, $2020^{35}$
1) $\mathrm{NaF}(2.5 \%)$ solutions with PEG-AgNPs at $12,800,6400,1600$, and $400 \mathrm{ppm}$ vs $38 \% \mathrm{SDF}$ (positive control) vs water(negative control)

2) Sample \& Distribution: Extracted sound human 3rd molars have been selected and 18 dentin slices were prepared for evaluation using a dental spectrophotometer.
PEG-AgNPs to NaF have not shown any staining effect, whereas SDF application resulted in obvious black staining.
Zhao IS et al, 202048
1) SDF vs NaF with PEG-AgNPs vs Deionized water(negative control)

2) Sample \& Distribution: Extracted sound human 3rd molars; 12 dentin blocks were allocated to each group for evaluation using a dental spectrophotometer.
Fluoridated PEG-AgNP does not stain dentin caries and hence it could be a better alternative to address the disadvantage of SDF.

SDF: Silver diamine Fluoride; NSF: Nanosilver fluoride; AgNPs: Silver nanoparticles; KF: Potassium fluoride; PEG-AgNPs: polyetbylene glycol-coated silver nanoparticles; NaF: Sodium fluoride.

\section{CONCLUSIONS}

NSF is a simple, inexpensive, non-toxic, noninvasive approach for arresting dental caries. It presents as an effective caries arresting agent that possess antibacterial actions of AgNPs and remineralizing properties of fluoride. It does not stain the carious lesion and hence showed to overcome the major limitation with SDF use.

\section{REFERENCES}

1. Bernabe E, Marcenes W, Hernandez CR, Bailey J, Abreu LG, et al. Global, regional, and national levels and trends in burden of oral conditions from 1990 to 2017: A Systematic Analysis for the Global Burden of Disease 2017 Study. J Dent Res 2020;99:362-373

2. Urquhart O, Tampi MP, Pilcher L, Slayton RL, Araujo MWB, Fontana M, et al. Nonrestorative treatments for caries: systematic review and network meta-analysis. J Dent Res 2019;98:14-26 
3. Slayton RL, Urquhart O, Araujo MWB, Fontana M, Guzmán-Armstrong S, Nascimento $\mathrm{MM}$, et al. Evidence-based clinical practice guideline on nonrestorative treatments for carious lesions: A report from the American Dental Association. J Am Dent Assoc 2018;149:837-849

4. Dorri M, Dunne SM, Walsh T, Schwendicke F. Micro-invasive interventions for managing proximal dental decay in primary and permanent teeth. Cochrane Database Syst Rev 2015;5:CD010431

5. Hendre AD, Taylor GW, Chávez EM, Hyde S. A systematic review of silver diamine fluoride: Effectiveness and application in older adults. Gerodontology 2017;34:411-419

6. Llodra JC, Rodriguez A, Ferrer B, Menardia V, Ramos T, Morato M. Efficacy of silver diamine fluoride for caries reduction in primary teeth and first permanent molars of schoolchildren: 36month clinical trial. J Dent Res 2005;84:721-734

7. Oliveira BH, Rajendra A, Veitz-Keenan A, Niederman R. The Effect of Silver Diamine Fluoride in Preventing Caries in the Primary Dentition: A Systematic Review and MetaAnalysis. Caries Res 2019;53:24-32

8. Subbiah GK, Gopinathan NM. Is silver diamine fluoride effective in preventing and arresting caries in elderly adults? A systematic review. J Int Soc Prev Community Dent 2018;8:191-199

9. Fakhruddin KS, Egusa H, Ngo HC, Panduwawala C, Pesee S, Venkatachalam T, et al. Silver diamine fluoride (SDF) used in childhood caries management has potent antifungal activity against oral Candida species. BMC Microbiol 2020;20;95-105

10. Karched M, Ali D, Ngo H. In vivo antimicrobial activity of silver diammine fluoride on carious lesions in dentin. J Oral Sci 2019;61:19-24

11. Mei ML, Li Q-L, Chu C-H, Lo EC-M, Samaranayake LP. Antibacterial effects of silver diamine fluoride on multi-species cariogenic biofilm on caries. Ann Clin Microbiol Antimicrob 2013;26;12:4

12. Fakhruddin KS, Egusa H, Ngo HC, Panduwawala C, Pesee S, Samaranayake LP. Clinical efficacy and the antimicrobial potential of silver formulations in arresting dental caries: a systematic review. BMC Oral Health 2020;20:160170
13. Zhao IS, Gao SS, Hiraishi N, Burrow MF, Duangthip D, Mei ML, et al. Mechanisms of silver diamine fluoride on arresting caries: a literature review. Int Dent J 2018;68:67-76

14. Punyanirun K, Yospiboonwong $T$, Kunapinun T, Thanyasrisung P, Trairatvorakul C. Silver diamine fluoride remineralized artificial incipient caries in permanent teeth after bacterial pH-cycling in-vitro. J Dent 2018;69:55-59

15. Yu OY, Zhao IS, Mei ML, Lo ECM, Chu CH. Caries-arresting effects of silver diamine fluoride and sodium fluoride on dentine caries lesions. J Dent 2018;78:65-71

16. Mei ML, Ito L, Cao Y, Li QL, Lo ECM, Chu $\mathrm{CH}$. Inhibitory effect of silver diamine fluoride on dentine demineralisation and collagen degradation. J Dent 2013;41:809-817

17. Primus C. Potassium Iodide. The Solution to Silver Diamine Fluoride Discoloration? Adv Dent Oral Health 2017;5:555655

18. Patel J, Anthonappa RP, King NM. Evaluation of the staining potential of silver diamine fluoride: in vitro. Int $\mathrm{J}$ Paediatr Dent 2018;28;514-522

19. Crystal YO, Kreider B, Raveis VH. Parental expressed concerns about silver diamine fluoride (sdf) treatment. J Clin Pediatr Dent $2019 ; 43: 155$ 160

20. Crystal YO, Janal MN, Hamilton DS, Niederman R. Parental perceptions and acceptance of silver diamine fluoride staining. J Am Dent Assoc 2017;148:510-518

21. Alshammari AF, Almuqrin AA, Aldakhil AM, Alshammari BH, Lopez JNJ. Parental perceptions and acceptance of silver diamine fluoride treatment in Kingdom of Saudi Arabia. Int J Health Sci 2019;13:25-29

22. Horst JA, Ellenikiotis H, Milgrom PM. UCSF Protocol for caries arrest using silver diamine fluoride: rationale, indications and consent. Pedia Dent 2017;84:16-26

23. Rosenblatt A, Stamford TCM, Niederman R. Silver diamine fluoride: a caries "silver-fluoride bullet." J Dent Res 2009;88:116-125

24. Zhao IS, Mei ML, Burrow MF, Lo EC-M, Chu C-H. Effect of silver diamine fluoride and potassium 1odide treatment on secondary caries prevention and tooth discolouration in cervical glass 1onomer cement restoration. Int J Mol Sci 2017;18:340-345 
25. Sayed M, Matsui N, Hiraishi N, Nikaido T, Burrow MF, Tagami J. Effect of glutathione biomolecule on tooth discoloration associated with silver diammine fluoride. Int $\mathrm{J}$ Mol Sci 2018;9:1322-1330

26. Roberts A, Bradley J, Merkley S, Pachal T, Gopal JV, Sharma D. Does potassium iodide application following silver diamine fluoride reduce staining of tooth? A systematic review. Aust Dent J 2020;65:109-117.

27. Turton B, Horn R, Durward C. Caries arrest and lesion appearance using two different silver fluoride therapies with and without potassium iodide: 6-month results. Heliyon. 2020;6:e04287

28. Turton B, Horn R, Durward C. Caries arrest and lesion appearance using two different silver fluoride therapies on primary teeth with and without potassium iodide: 12 -month results. Clin Exp Dent Res 2020 Dec 02. Doi:10.1002/cre2.367

29. Espíndola-Castro LF, Rosenblatt A, Galembeck A, Monteiro G. Dentin staining caused by nano-silver fluoride: A comparative study. Oper Dent 2020;45:435-441

30. Sayed M, Hiraishi N, Matin K, Abdou A, Burrow MF, Tagami J. Effect of silver-containing agents on the ultra-structural morphology of dentinal collagen. Dent Mater 2020;36:936-944

31. Javan Bakht Dalir S, Djahaniani H, Nabati F, Hekmati M. Characterization and the evaluation of antimicrobial activities of silver nanoparticles biosynthesized from leaf extract. Heliyon 2020;6:e03624

32.Mikhailov OV, Mikhailova EO. Elemental silver nanoparticles: Biosynthesis and bio applications. Materials 2019;12:3177

33. Yin IX, Zhao IS, Mei ML, Li Q, Yu OY, Chu $\mathrm{CH}$. Use of silver nanomaterials for caries prevention: A concise review. Int J Nanomedicine 2020;15:3181-3191

34.Yin IX, Yu OY, Zhao IS, Mei ML, Li Q-L, Tang J, et al. Developing biocompatible silver nanoparticles using epigallocatechin gallate for dental use. Arch Oral Biol 2019;102:106-112

35. Yin IX, Zhao IS, Mei ML, Lo ECM, Tang J, $\mathrm{Li} Q$, et al. Synthesis and characterization of fluoridated silver nanoparticles and their potential as a non-staining anti-caries agent. Int $\mathrm{J}$ Nanomedicine 2020;15:3207-3215
36. Tirupathi S, Svsg N, Rajasekhar S, Nuvvula S. Comparative cariostatic efficacy of a novel Nanosilver fluoride varnish with $38 \%$ silver diamine fluoride varnish a double-blind randomized clinical trial. J Clin Exp Dent 2019;11:105-112

37. Burns J, Hollands K. Nano silver fluoride for preventing caries. Evid Based Dent 2015;16:8-9

38. Santos VE dos Jr, Vasconcelos Filho A, Targino AGR, Flores MAP, Galembeck A, Caldas AF Jr, et al. A new "silver-bullet" to treat caries in children--nano silver fluoride: a randomised clinical trial. J Dent 2014;42:945-951

39. Baker C, Pradhan A, Pakstis L, Pochan DJ, Shah SI. Synthesis and antibacterial properties of silver nanoparticles. J Nanosci Nanotechnol 2005;5:244-249

40. Morones JR, Elechiguerra JL, Camacho A, Holt K, Kouri JB, Ramírez JT, et al. The bactericidal effect of silver nanoparticles. Nanotechnology 2005;16:2346-2353

41. Targino AGR, Flores MAP, dos Santos Junior VE, de Godoy Bené Bezerra F, de Luna Freire H, Galembeck A, et al. An innovative approach to treating dental decay in children. A new anti-caries agent J Mater Sci Mater Med 2014;10: 2041-2047 42. Teixeira JA, Silva AVCE, Dos Santos Júnior VE, de Melo Júnior PC, Arnaud M, Lima MG, et al. Effects of a new nano-silver fluoridecontaining dentifrice on demineralization of enamel and adhesion and acidogenicity. Int J Dent 2018;2018:1351925

43. Silva AVC, Amitis Vieira Costa, Teixeira JA, Cláudia C B, Emery Clayton Cabral, de Melo Júnior PC, et al. In Vitro morphological, optical and microbiological evaluation of nanosilver fluoride in the remineralization of deciduous teeth enamel Nanotechnol Rev 2018;7:509-520

44. Freire PLL, Albuquerque AJR, Sampaio FC, Galembeck A, Flores MAP, Stamford TCM, et al. AgNPs: The new allies against s. mutans biofilm a pilot clinical trial and microbiological assay. Braz Dent J 2017;28:417-422

45. Silva AVC, Amitis Vieira Costa, de Araújo Teixeira J, de Melo Júnior PC, de Souza Lima MG, de Oliveira Mota CCB, et al. Remineralizing potential of nano-silver-fluoride for tooth enamel: An optical coherence tomography analysis. Pesqui Bras Odontopediatria Clin Integr 2019;19: 1-13

46. Nozari A, Ajami S, Rafiei A, Niazi E. Impact of nano hydroxyapatite, nano silver fluoride and 
sodium fluoride varnish on primary teeth enamel remineralization: An in vitro study. J Clin Diagn Res 2017;11:97-100

47. Akyildiz M, Sönmez IS. Comparison of remineralising potential of nano silver fluoride, silver diamine fluoride and sodium fluoride varnish on artificial caries: an in vitro study. Oral Health Prev Dent 2019;17:469-477

48. Zhao IS, Yin IX, Mei ML, Lo ECM, Tang J, $\mathrm{Li} \mathrm{Q}$, et al. Remineralising dentine caries using sodium fluoride with silver nanoparticles: An in vitro study. Int J Nanomedicine 2020;15:2829_ 2839

49. Chu CH, Lo ECM, Lin HC. Effectiveness of silver diamine fluoride and sodium fluoride varnish in arresting dentin caries in Chinese preschool children. J Dent Res 2002;81:767-770

50. Nagireddy VR, Reddy D, Kondamadugu S, Puppala N, Mareddy A, Chris A. Nanosilver fluoride-A paradigm shift for arrest in dental caries in primary teeth of schoolchildren: A randomized controlled clinical trial. Int J Clin Pediatr Dent 2019;12:484-490

51. Westland S, Luo W, Li Y, Pan Q, Joiner A. Investigation of the perceptual thresholds of tooth whiteness. J Dent 2017;67:11-14

52. Zander HA. use of silver nitrate in the treatment of caries. J Am Dent Assoc 1941;28:1260-1267

53. Crystal YO, Niederman R. Evidence-based dentistry update on silver diamine fluoride. Dent Clin North Am 2019;63:45-68

54. Köhler W. WD Miller. The micro-organisms of the human mouth 1974;14:84-84

55. Muntz JA, Dorfman A, Stephan RM. In vitro studies on sterilization of carious dentin. Evaluation of germicides. J Am Dent Assoc 1943;30:1893-900

56. Gao SS, Zhao IS, Duffin S, Duangthip D, Lo $\mathrm{ECM}$, Chu $\mathrm{CH}$. Revitalising silver nitrate for caries management. Int $\mathrm{J}$ Environ Res Public Health 2018;15:80-85

57. Yamaga R, Nishino M, Yoshida S, Yokomizo

I. Diammine silver fluoride and its clinical application. J Osaka Univ Dent Sch 1972;12:1-20

58. Thibodeau EA, Handelman SL, Marquis RE. Inhibition and killing of oral bacteria by silver 1ons generated with low intensity direct current. J Dent Res 1978;57:922-926
59. Craig GG, Powell KR, Cooper MH. Caries progression in primary molars: 24-month results from a minimal treatment programme. Community Dent Oral Epidemiol 1981;9:260 265

60. Craig GG, Powell KR, Cooper MH. Clinical appearance of permanent successors after nonextraction treatment of grossly carious primary molars in highly anxious children. ASDC J Dent Child 1987;54:170-175

61. Green E. A clinical evaluation of two methods of caries prevention in newly-erupted first permanent molars. Aust Dent J 1989;34: 407-419 62. Yee R, Holmgren C, Mulder J, Lama D, Walker D, van Palenstein Helderman W. Efficacy of silver diamine fluoride for arresting caries treatment. J Dent Res 2009;88:644-657

63. Zhi QH, Lo ECM, Lin HC. Randomized clinical trial on effectiveness of silver diamine fluoride and glass ionomer in arresting dentine caries in preschool children. J Dent 2012;40:962967

64. Zhang W, McGrath C, Lo ECM, Li JY. Silver diamine fluoride and education to prevent and arrest root caries among community-dwelling elders. Caries Res. 2013;47:284-290

65. Dos Santos VE Jr, de Vasconcelos FMN, Ribeiro AG, Rosenblatt A. Paradigm shift in the effective treatment of caries in schoolchildren at risk. Int Dent J 2012;62:47-51

66. Jabin Z, Vishnupriya V, Agarwal N, Nasim I, Jain M, Sharma A. Effect of 38\% silver diamine fluoride on control of dental caries in primary dentition: A systematic review. J Family Med Prim Care 2020;9:1302-1307

67. de Almeida L de FD, Cavalcanti YW, Valença AMG. In vitro antibacterial activity of silver diamine fluoride in different concentrations. Acta Odontol Latinoam 2011;24:127-131

68. Mei ML, Ito L, Cao Y, Lo ECM, Li QL, Chu $\mathrm{CH}$. An ex vivo study of arrested primary teeth caries with silver diamine fluoride therapy. J Dent 2014;42:395-402

69. Chu C-H, Lee AH-C, Zheng L, Mei ML, Chan GC-F. Arresting rampant dental caries with silver diamine fluoride in a young teenager suffering from chronic oral graft versus host disease postbone marrow transplantation: A case report. BMC Res Notes 2014:7:3-10 
70. Corrêa JM, Mori M, Sanches HL, da Cruz AD, Poiate E, Isis Andréa Venturini. Silver Nanoparticles in Dental Biomaterials. Int J Biomater 2015;1:1-9

\section{How to cite this article:}

Mohammed Zameer, Sameen Badiujjama Birajdar, Syed Wali Peeran, Syed Nahid Basheer, Syed Ali Peeran, and Arun Reddy. Nanosilver fluoride as a caries arresting agent: A narrative review. Contemp Pediatr Dent 2021:2(1):1-13

\section{Declarations}

Acknowledgements: Not applicable.

Conflict of Interest Statement: The authors disclose no potential conflicts of interest.

Ethics Statement: This study does not require approval from the ethics committee.

Informed Consent: Not required.

Author contributions: Conception and design: $M Z$; Acquisition of data: MZ, SNB; Interpretation of data: $M Z$, SWP; Drafting article: $M Z, S B B, A R$; Revision artice: SBB, SNB, SAP, AR; Final approval: All Authors

Funding: This work is not finantiated.

Data Availability: The data used to support the findings of this study can be made available upon request to the corresponding author.

Peer-review: Externally double-blinded peer-reviewed. 